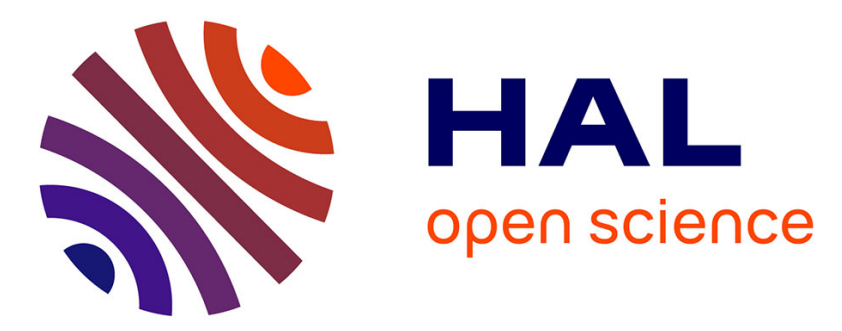

\title{
Circuit realization method for reduced order inductive PEEC modeling circuits
}

Trung-Son Nguyen, Tung Le Duc, Son Thanh Tran, Jean-Michel Guichon, Olivier Chadebec

\section{- To cite this version:}

Trung-Son Nguyen, Tung Le Duc, Son Thanh Tran, Jean-Michel Guichon, Olivier Chadebec. Circuit realization method for reduced order inductive PEEC modeling circuits. COMPEL: The International Journal for Computation and Mathematics in Electrical and Electronic Engineering, 2016, 35 (3), pp.1203-1217. 10.1108/COMPEL-11-2015-0425 . hal-02277757

\section{HAL Id: hal-02277757 \\ https://hal.science/hal-02277757}

Submitted on 15 Dec 2020

HAL is a multi-disciplinary open access archive for the deposit and dissemination of scientific research documents, whether they are published or not. The documents may come from teaching and research institutions in France or abroad, or from public or private research centers.
L'archive ouverte pluridisciplinaire HAL, est destinée au dépôt et à la diffusion de documents scientifiques de niveau recherche, publiés ou non, émanant des établissements d'enseignement et de recherche français ou étrangers, des laboratoires publics ou privés. 


\title{
CIRCUIT REALIZATION METHOD FOR REDUCED ORDER INDUC- TIVE PEEC MODELING CIRCUITS
}

\author{
Trung-Son Nguyen, Tung Le Duc, Son Thanh Tran, Jean-Michel Guichon, Olivier Chadebec
}

\begin{abstract}
Purpose - To synthesize equivalent circuit obtained from reduced order model of large scale inductive PEEC circuits.

Design/methodology/approach -This paper describes an original approach for reducing and synthesizing large parasitic RLM electrical circuits coming from inductive Partial Element Equivalent Circuit (PEEC) models. The proposed technique enables the re-use of the reduced order model in the time domain circuit simulation context.

Findings - The paper shows how to use a synthesis method to realize an equivalent circuit issued from compressed PEEC circuits.

Originality/value - The coupling between methods PEEC and a compressed method as Fast Multipole Method (FMM) in order to reduce time and space consuming are well-known. The innovation here is to realize a smaller circuit equivalent with the original large scale PEEC circuits to use in temporal simulation tools. Moreover, this synthesis method reduces time and memories for modelling industrial application while maintaining high accuracy.
\end{abstract}

Keywords Interconnect circuit, model order reduction, equivalent circuit synthesis method, PEEC method, FMM method, SPRIM, IOPOR.

Paper type Research paper

\section{Introduction}

PARTIAL ELEMENT EQUIVALENT CIRCUIT (PEEC) approach (Ruehli, 1974) is known to be very suitable for the modelling of power electronic devices with complex set of conductors. Using the PEEC method, the electromagnetic behaviour of arbitrarily 3D conducting devices can be represented by equivalent circuits combining resistors, partial/mutual inductances (computed numerically thanks to an integral formulation). As the problem is transferred from the electromagnetic domain to the circuit domain (Wollenberg et al., 2011), any conventional SPICE-like circuit solvers can be employed to analyze the equivalent circuit and other electrical components such as current and/or voltage sources, resistor, capacitor and inductance could be easily included to the PEEC model. In many applications within power electronics, the magnetic field is a dominating factor over the electric field due to the high current in the systems, therefore internal capacitive effects could be neglected and the method is restricted to its inductive formulation.

However, in high complex industrial applications, the increment of number of conductor elements (directly proportional with the complexity of the devices) causes an explosion in memory by the number of terms in the full PEEC matrix. In addition, the equivalent circuit is very large and each mutual inductances issue of PEEC model is coupled with other (fully dense matrices) so it's rapidly impossible to simulate a power electronic device in a temporal simulation tool (like PSpice, Saber...) with an electrical scheme derived from PEEC approach because of a high number of elementary electrical circuit elements.

In order to solve the problem of fully dense matrix (saving memory and computation time), we treat the interactions between far-away basis functions by the Fast Multipole Method (FMM) (Ardon et al., 2010; Zhang et al., 2014). The corresponding matrix elements do not need to be explicitly stored, resulting in a significant reduction of memory requirements. In consequence, by using the FMM, the resulted model is available only in the frequency domain and the link with a conventional circuit representation as the traditional PEEC method is lost. To be included in time domain simulation of circuit-solvers, this model needs to be replaced by a reducedorder model, which is much smaller but would produce a very good approximation of input-output behaviour of the original device.

This problem can be solved in two steps. The first is to reduce the size of PEEC frequency model by using an appropriate model order reduction method. We then obtained a simpler and lighter frequency model to handle. In the second step, the reduced model is synthesized into an equivalent circuit as a netlist representation (RLC elements), which can be integrated in a SPICE-like circuit-solver. The pure RLC model is then simple enough to ensure the convergence of the resolution and accurate enough to return acceptable results. In this paper, we investigate the second step: the circuit realization method from the reduction of frequency model.

In the first step, the use of matrix compression algorithm like Fast Multipole Method (FMM) leads to the restriction on algebraic operation: only product matrix/vector is allowed. Due to this context, only Model Order Reduction (MOR) method based on projection approach is suitable. These popular methods of this kind such as Passive Reduced-order Interconnect Macro-modeling Algorithm (PRIMA) (Odabasioglu et al., 1997), Structurepreserving Reduced-order Interconnect Macro-modeling (SPRIM) (Freund, 2004) are well known to generate a good Reduced-order Model (ROM) while the stability of the reduced system is preserved. However, the ROM obtained by these two approaches are in the state space form which is not compatible to import into a temporal simulation tools because lots of temporal simulation tools accept only a netlist representation (RLC elements) as 
input. To overcome this issue, the authors (Yang et al, 2008) proposed an embeddable Input-Output structure Preserving Order Reduction (IOPOR) technique to further preserve the structures of input and output incidence matrices. This technique is the combination of block structure preserving MOR methods and IOPOR techniques (SPRIM/IOPOR) which guarantee pure RLC equivalent circuits can be synthesized. The authors also presented an RLC equivalent circuit synthesis method RLCSYN (RLC SYN-thesis). However, the RLCSYN approach (Ionutiu et al, 2009; Kaufmann, 2010) cannot synthesize the mutual inductances issued from the inductive PEEC approach.

Considering these difficulties, this paper presents an original approach to synthesis the electric circuits issued roman appropriate MOR projection method (SPRIM/IOPOR). The final reduced circuit will be presented as a netlist of RLC elements. This article is composed of three parts:

- in the first part, a summary of the classical PRIMA method is presented, following by a variant of the SPRIM/IOPOR method;

- in the second part, an original circuit synthesis method is proposed to obtain a reduced electrical circuit outcome from the SPRIM/IOPOR method in the first part;

- finally in the last part, an example of EMC filter modelling is presented to validate our methodology.

\section{Background}

\subsection{PEEC RLMC circuit equations}

Considering a RLMC circuit that is only excited by current sources. The incidence matrix of nodes / branches $\mathbf{A}$, the branch currents vector $\mathbf{i}_{\mathrm{B}}$ and nodal voltages vector $\mathbf{v}_{\mathrm{N}}$ can be written as follows:

$$
\mathbf{A}=\left[\begin{array}{llll}
\mathbf{A}_{\mathrm{R}} & \mathbf{A}_{\mathrm{C}} & \mathbf{A}_{\mathrm{L}} & \mathbf{A}_{\mathrm{I}}
\end{array}\right] ; \mathbf{i}_{\mathrm{B}}=\left[\begin{array}{llll}
\mathbf{i}_{\mathrm{R}} & \mathbf{i}_{\mathrm{C}} & \mathbf{i}_{\mathrm{L}} & \mathbf{i}_{\mathrm{I}}
\end{array}\right]^{T} ; \mathbf{v}_{\mathrm{N}}=\left[\begin{array}{llll}
\mathbf{v}_{\mathrm{R}} & \mathbf{v}_{\mathrm{C}} & \mathbf{v}_{\mathrm{L}} & \mathbf{v}_{\mathrm{I}}
\end{array}\right]^{T}
$$

where the subscripts $\mathrm{R}, \mathrm{C}, \mathrm{L}$ and I are associated with the branches containing resistors, the external capacitors, partial inductors (calculated by the PEEC integral method (Clavel et al, 2002)), the external inductances and the external current sources. The branch constitutive relations of circuit elements provide:

$$
\mathbf{i}_{\mathrm{I}}=-\mathbf{I}_{\mathrm{s}} ; \mathbf{i}_{\mathrm{R}}=\mathbf{R}_{\mathrm{EXT}}{ }^{-1} \cdot \mathbf{v}_{\mathrm{R}} ; \mathbf{i}_{\mathrm{C}}=\mathbf{C}_{\mathrm{EXT}} \frac{d}{d t} \mathbf{v}_{\mathrm{C}} ; \mathbf{v}_{\mathrm{L}}=\mathbf{L}_{\mathrm{EXT}} \frac{d}{d t} \mathbf{i}_{\mathrm{L}}
$$

where $\mathbf{I}_{\mathrm{s}}$ is the vector of current sources, $\mathbf{R}_{\mathrm{EXT}}$ and $\mathbf{C}_{\mathrm{EXT}}$ are diagonal matrices and $\mathbf{L}_{\mathrm{EXT}}$ is the inductance matrix from the external circuit. In the case of the full inductive coupling PEEC matrix $\mathbf{L}_{\mathrm{PEEC}}$ and diagonal resistance matrix $\mathbf{R}_{\mathrm{PEEC}}$, we have the equation restricted to the PEEC elements:

$$
\mathbf{v}_{\text {IPEEC }}=\mathbf{R}_{\mathrm{PEEC}} \cdot \mathbf{i}_{\text {IPEEC }}+\mathbf{L}_{\mathrm{PEEC}} \frac{d}{d t} \mathbf{i}_{\text {IPEEC }}
$$

The modified nodal analysis (MNA) (Chung-Wen et al, 1975) has been widely used for formulating circuit equations for the PEEC Method (Ekman et al, 2004; Freund, 2004). The descriptor system is presented in the form:

$$
\left\{\begin{array}{l}
\mathbf{E} \frac{d \mathbf{x}(t)}{d t}=-\mathbf{G} \cdot \mathbf{x}(t)+\mathbf{B} \cdot \mathbf{u}(t) \\
\mathbf{y}(t)=\mathbf{B}^{\mathbf{T}} \cdot \mathbf{x}(t)
\end{array}\right.
$$

where

$$
\begin{aligned}
& \mathbf{E}=\left[\begin{array}{c}
\mathbf{A}_{\mathrm{C}} \cdot \mathbf{C}_{\mathrm{EXT}} \cdot \mathbf{A}_{\mathrm{C}}^{T} \\
0 \\
0
\end{array} \quad\left[\begin{array}{cc}
\mathbf{L}_{\mathrm{PEEC}} & 0 \\
0 & \mathbf{L}_{\mathrm{EXT}}
\end{array}\right]\right]=\left[\begin{array}{cc}
\mathbf{E}_{1} & 0 \\
0 & \mathbf{E}_{2}
\end{array}\right] ; \\
& \mathbf{G}=\left[\begin{array}{c}
\mathbf{A}_{\mathrm{R}} \cdot \mathbf{R}_{\mathrm{EXT}}{ }^{-1} \cdot \mathbf{A}_{\mathrm{R}}^{T} \\
-\mathbf{A}_{\mathrm{L}}^{T}
\end{array} \quad\left[\begin{array}{cc}
\mathbf{R}_{\mathrm{LEEC}} & 0 \\
0 & 0
\end{array}\right]\right]=\left[\begin{array}{cc}
\mathbf{G}_{1} & \mathbf{A}_{\mathrm{L}} \\
-\mathbf{A}_{\mathrm{L}}^{T} & \mathbf{G}_{2}
\end{array}\right] ; \\
& \mathbf{B}=\left[\begin{array}{c}
\mathbf{A}_{\mathrm{I}} \\
0
\end{array}\right] ; \quad \mathbf{x}=\left[\begin{array}{c}
\mathbf{v}_{\mathrm{N}} \\
\mathbf{i}_{\mathrm{L}}
\end{array}\right] ; \mathbf{u}=-\mathbf{i}_{\mathrm{I}} ;
\end{aligned}
$$

By applying the Laplace transform from a function of a positive real variable $t$ (time) to a function of a complex variable $s$ (frequency) for (4), the impedance matrix $\mathbf{Z}(s)$ or transfer function of the descriptor system is calculated:

$$
\mathbf{Z}(s)=\mathbf{B}^{T} \cdot(\mathbf{G}+s \cdot \mathbf{E})^{-1} \cdot \mathbf{B}
$$

The purpose of model order reduction method is to find a reduced model having the same form as the original one in (4) which can be written by the descriptor form in (7a) or in the Laplace transform in (7b): 


$$
\begin{gathered}
\left\{\begin{array}{l}
\hat{\mathbf{E}} \frac{d \mathbf{z}(t)}{d t}=-\hat{\mathbf{G}} \cdot \mathbf{z}(t)+\hat{\mathbf{B}} \cdot \mathbf{u}(t) \\
\mathbf{y}(t)=\hat{\mathbf{B}}^{\mathbf{T}} \cdot \mathbf{z}(t)
\end{array}\right. \\
\left\{\begin{array}{l}
s \cdot \hat{\mathbf{E}} \cdot \mathbf{z}(s)=-\hat{\mathbf{G}} \cdot \mathbf{z}(s)+\hat{\mathbf{B}} \cdot \mathbf{u}(s) \\
\mathbf{y}(s)=\hat{\mathbf{B}}^{\mathrm{T}} \cdot \mathbf{z}(s)
\end{array}\right.
\end{gathered}
$$

The most important property of MOR approach is the impedance matrix of new reduced model must be approximate the original one. In the next section, we present our MOR method. From this section, the descriptor system is always written in the Laplace transform.

\subsection{PEEC RLMC circuit equations}

With an expansion point $s_{0}$, we can rewrite equation (6) as follows:

and

$$
\mathbf{Z}(s)=\mathbf{B}^{T} \cdot\left(\mathbf{G}+s_{0} \cdot \mathbf{E}+\left(s-s_{0}\right) \cdot \mathbf{E}\right)^{-1} \cdot \mathbf{B}=\mathbf{B}^{T} \cdot\left(\mathbf{I}-\left(s-s_{0}\right) \cdot \mathbf{D}\right)^{-1} \cdot \mathbf{F}
$$

$$
\mathbf{D}=-\left(\mathbf{G}+s_{0} \cdot \mathbf{E}\right)^{-1} \cdot \mathbf{E} ; \mathbf{F}=\left(\mathbf{G}+s_{0} \cdot \mathbf{E}\right)^{-1} \cdot \mathbf{B}
$$

In the conventional PRIMA method (Odabasioglu et al., 1997), only one expansion point $(s=0)$ is used to construct the Krylov subspace (by the block Arnoldi algorithm). This approach is equivalent to a Taylor series expansion of the last equation (8) at $s_{0}=0$.

In our approach, several expansion points are selected for the construction of Krylov subspace. The multipoint approximants are usually more expensive to construct than single point interpolation as PRIMA, but they tend to have better quality models for given effort (Wolf et al., 2011). Multi-point based approaches (Feng et al., 2012; Villena et al., 2009; Silveira et al., 2006; Ferranti et al., 2011; Rasekh et al., 2012) have recently gathered renewed attention due to their robustness, reliability.

Combination of several subspaces for each expansion point $s_{j}$ gives us the new Krylov subspace $\mathbf{U}_{m}$ :

$$
\mathbf{U}_{m}=\operatorname{span}\{\underbrace{\mathbf{F}, \mathbf{D} \cdot \mathbf{F}, \mathbf{D}^{2} \cdot \mathbf{F}, \ldots, \mathbf{D}^{q-1} \cdot \mathbf{F}}_{\text {point } s=0}, \underbrace{\ldots \mathbf{F}_{j}, \mathbf{D}_{j} \cdot \mathbf{F}_{j}, \mathbf{D}_{j}{ }^{2} \cdot \mathbf{F}_{j}, \ldots, \mathbf{D}_{j}{ }^{q-1} \cdot \mathbf{F}_{j}}_{\text {point } s \neq 0}\}=\operatorname{span}\left\{s_{0}, s_{1}, \ldots, s_{j}\right\}
$$

The Krylov subspace therefore consists of a first portion corresponding to the first point of expansion and a second portion corresponding to the second point and so on. Let's notice that the vectors constituting this subspace are orthogonal with each other so the blocks representing different points of expansion are orthogonal.

The main computational cost of this method is computing matrix-vector product ( $\mathbf{D} \cdot \mathbf{x}$ in the following) in the block Arnoldi process to obtain $\mathbf{U}_{m}$.

With the expansion point $s=0, \mathbf{D} \cdot \mathbf{x}$ is the product made by solving a linear system:

$$
\mathbf{D} \cdot \mathbf{x}=\mathbf{G}^{-1} \cdot \mathbf{E} \cdot \mathbf{x}=\mathbf{v} \Leftrightarrow \mathbf{G} \cdot \mathbf{v}=\mathbf{E} \cdot \mathbf{x}
$$

The matrix $\mathbf{G}$ is a sparse matrix (which incorporates mainly the resistance); we use a sparse direct LU solver with AMD algorithm (Davis, 2006) to reorganize matrix for conserving a sparse LU decomposition which enhance then overall performance. In the case of circuits obtained with PEEC, the matrix $\mathbf{E}$ (especially $\mathbf{L}_{\mathrm{PEEC}}$ ) is compressed by an algorithm of type FMM (Ardon et al., 2010; Nguyen et al., 2012) so the matrix-vector product $\mathbf{E} \cdot \mathbf{x}$ is greatly accelerated from $O\left(N^{2}\right)$ to $O(N \log N)$ by FMM (N is the size of matrix $\left.\mathbf{D}\right)$.

With the expansion point $s \neq 0$, we have a new linear system:

$$
\mathbf{D} \cdot \mathbf{x}=\left(\mathbf{G}+s_{0} \cdot \mathbf{E}\right)^{-1} \cdot \mathbf{E} \cdot \mathbf{x}=\mathbf{v} \Leftrightarrow\left(\mathbf{G}+s_{0} \cdot \mathbf{E}\right) \cdot \mathbf{v}=\mathbf{E} \cdot \mathbf{x}
$$

Note that $\left(\mathbf{G}+s_{0} \cdot \mathbf{E}\right)$ is compressed by FMM, so that the system (12) is no more sparse. To solve (12), we need a solver compatible with FMM. In this case, iterative solver like GMRES is chosen by its efficiency.

After the construction of Krylov subspace, the reduced matrices obtained with the congruent transformation of PRIMA are then:

$$
\hat{\mathbf{E}}=\mathbf{U}_{m}^{T} \cdot \mathbf{E} \cdot \mathbf{U}_{m} ; \hat{\mathbf{G}}=\mathbf{U}_{m}^{T} \cdot \mathbf{G} \cdot \mathbf{U}_{m} ; \hat{\mathbf{B}}=\mathbf{U}_{m}^{T} \cdot \mathbf{B}
$$

The transfer function $\mathbf{Z}_{\mathrm{R}}(s)$ is then reduced:

$$
\mathbf{Z}_{\mathrm{R}}(s)=\hat{\mathbf{B}}^{\mathrm{T}} \cdot(\hat{\mathbf{G}}+s \cdot \hat{\mathbf{E}})^{-1} \cdot \hat{\mathbf{B}}
$$

All the important properties of the PRIMA method like the preservation of passivity and the preservation of moments are conserved.

If the basis matrix can be partitioned as $\mathbf{U}_{m}=\left[\mathbf{U}_{1} \mathbf{U}_{2}\right]^{\mathrm{T}}$ where the number of row of $\mathbf{U}_{1}, \mathbf{U}_{2}$ correspond respectively to $\mathbf{E}_{\mathbf{1}}, \mathbf{E}_{\mathbf{2}}$ (cf. 5a) then the new reduced unknown is now $\mathbf{z}(s)$ where: 


$$
\begin{aligned}
& \mathbf{z}(s)=\mathbf{U}_{m} \cdot \mathbf{x}(s) \\
& \mathbf{z}(s)=\mathbf{U}_{1} \cdot \mathbf{v}_{\mathrm{N}}(s)+\mathbf{U}_{2} \cdot \mathbf{i}_{\mathrm{L}}(s)
\end{aligned}
$$

Then we can rewrite new reduced circuit equations in frequency domain (Laplace transform):

$$
\left\{\begin{array}{l}
(s \cdot \hat{\mathbf{E}}+\hat{\mathbf{G}}) \cdot \mathbf{z}(s)=\hat{\mathbf{B}} \cdot \mathbf{u}(s) \\
\mathbf{y}(s)=\hat{\mathbf{B}}^{T} \cdot \mathbf{z}(s)
\end{array}\right.
$$

The reduced model in (7b) is then achieved in (16). However, in (15) the unknown $\mathbf{z}(s)$ is the sum of voltage and current and it cannot be expressed as a basic element circuit. Therefore, the realization of this equation to a circuit is impossible. In addition, the reduced incidence matrix $\hat{B}$ loses the property of original incidence matrix because in (13) it has some values different than $-1,0,1$. The reduced model issued from the PRIMA method is impossible to be synthesized as equivalent circuits.

In the next section, we present the SPRIM/IOPOR model reduction method to preserve some block structure and input/output behaviour which facility the synthesis of reduced system.

\subsection{SPRIM/IOPOR for PEEC circuits}

In this section, we developed a method based on SPRIM/IOPOR approaches (Freund, 2004; Yang et al, 2008). The algorithm enables the preservation of block nature (state variables natures) and input/output incidence matrices to guarantee the pure RLC equivalent circuits can be synthesized.

The block structure-preserving technique SPRIM (Freund, 2004) employs the basis matrix $\mathbf{U}_{\mathrm{m}}=\left[\mathbf{U}_{1} \mathbf{U}_{2}\right]^{\mathrm{T}}$ of size $\mathrm{N} \times \mathrm{m}$ created by well-known block Arnoldi algorithm from PRIMA method in section 2.2 ( $\mathrm{N}$ is the size of original matrix D). This matrix is constructed from Krylov subspace vectors spanned by the first $q$ block moments of the state variables in (10) for each expansion point. Instead of using $\mathbf{U}_{m}$ as the projection matrix, a larger orthonormal matrix $\left[\begin{array}{cc}\mathbf{U}_{1} & 0 \\ 0 & \mathbf{U}_{2}\end{array}\right]$ is employed to project the original system (4) to new reduced model as:

$$
\begin{gathered}
\hat{\mathbf{E}}_{\text {SPRIM }}=\left[\begin{array}{cc}
\mathbf{U}_{1}^{T} \cdot \mathbf{E}_{1} \cdot \mathbf{U}_{1} & 0 \\
0 & \mathbf{U}_{2}^{T} \cdot \mathbf{E}_{2} \cdot \mathbf{U}_{2}
\end{array}\right] ; \hat{\mathbf{G}}_{\text {SPRIM }}=\left[\begin{array}{cc}
\mathbf{U}_{1}^{T} \cdot \mathbf{G}_{1} \cdot \mathbf{U}_{1} & \mathbf{U}_{1}^{T} \cdot \mathbf{A}_{\mathrm{L}} \cdot \mathbf{U}_{2} \\
-\mathbf{U}_{2}^{T} \cdot \mathbf{A}_{\mathrm{L}}^{T} \cdot \mathbf{U}_{1} & \mathbf{U}_{2}^{T} \cdot \mathbf{G}_{2} \cdot \mathbf{U}_{2}
\end{array}\right] \\
\hat{\mathbf{B}}_{\text {SPRIM }}=\left[\begin{array}{c}
\mathbf{U}_{1}^{T} \cdot \mathbf{A}_{\mathrm{I}} \\
0
\end{array}\right]
\end{gathered}
$$

Now the reduced unknowns preserved their original natures (voltages or currents):

$$
\left[\begin{array}{l}
\mathbf{v}_{\text {N_SPRIM }}(s) \\
\mathbf{i}_{\text {L_SPRIM }}(s)
\end{array}\right]=\left[\begin{array}{l}
\mathbf{U}_{1} \cdot \mathbf{v}_{\mathrm{N}}(s) \\
\mathbf{U}_{2} \cdot \mathbf{i}_{\mathrm{L}}(s)
\end{array}\right]
$$

However, the structure of input and output (I/O) is still not preserved because in the equation (18) the new reduced incidence matrix can have some elements differ from $-1,0,1$.

In order to preserve the I/O structure of original system in its reduced-order model, we need to modify the matrix $\mathbf{U}_{1}$ by IOPOR method (Yang et al, 2008).

For a o-input and p-output reduced RLMC interconnect network with $\mathrm{N}_{\mathrm{S}}$ current sources, $\mathrm{N}_{\mathrm{ES}}$ number of input-output, we have $\mathrm{N}_{\mathrm{N}}$ node voltages and $\mathrm{N}_{\mathrm{L}}$ currents through the inductors (see (19)), the total number of unknown is $\mathrm{N}_{\mathrm{N}}+\mathrm{N}_{\mathrm{L}}=\mathrm{N}$. The incidence matrix $\mathbf{A}_{\mathrm{I}}$ can be described as $\mathbf{A}_{\mathrm{I}}=\underbrace{\left[\begin{array}{c}\mathbf{A}_{\mathrm{IN}} \\ 0_{\mathrm{N}_{\mathrm{N}}-\mathrm{N}_{\mathrm{ES}}}\end{array}\right]}_{\mathbf{N}_{\mathrm{S}}}$ and the basis matrix $\mathbf{U}_{\mathbf{1}}$ is $\mathbf{U}_{1}=\underbrace{\left[\begin{array}{c}\mathbf{U}_{1 \mathrm{~N}_{\mathrm{ES}}} \\ \mathbf{U}_{1\left(\mathrm{~N}_{\mathrm{N}}-\mathrm{N}_{\mathrm{ES}}\right)}\end{array}\right]}_{m}$

We then modify $\mathbf{U}_{1}$ as follow:

$$
\tilde{\mathbf{U}}_{1}=\underbrace{\left[\begin{array}{cc}
\mathbf{I d}_{\mathrm{N}_{\mathrm{ES}} \mathrm{N}_{\mathrm{ES}}} & 0 \\
0 & \mathbf{U}_{1\left(\mathrm{~N}_{\mathrm{N}}-\mathrm{N}_{\mathrm{ES}}\right)}
\end{array}\right]}_{m+N_{E S}}
$$

So the product $\tilde{\mathbf{U}}_{1}^{T} \cdot \mathbf{A}_{\mathrm{I}}$ conserve the structure:

$$
\tilde{\mathbf{U}}_{1}^{T} \cdot \mathbf{A}_{\mathrm{I}}=\left[\begin{array}{cc}
\mathbf{I d}_{\mathrm{N}_{\mathrm{ES} N \mathrm{NS}}} & 0 \\
0 & \mathbf{U}_{1\left(\mathrm{~N}_{\mathrm{L}}-\mathrm{N}_{\mathrm{ES}}\right)}^{T}
\end{array}\right] \cdot\left[\begin{array}{c}
\mathbf{A}_{\mathrm{IN} \mathrm{ES}} \\
0_{\mathrm{N}_{\mathrm{L}}-\mathrm{N}_{\mathrm{ES}}}
\end{array}\right]=\left[\begin{array}{c}
\mathbf{A}_{\mathrm{IN}_{\mathrm{ES}}} \\
0_{\mathrm{N}_{\mathrm{L}}-\mathrm{N}_{\mathrm{ES}}}
\end{array}\right]
$$

We have the matrices of new reduced system 


$$
\begin{gathered}
\hat{\mathbf{E}}_{\text {SPRIM_IOPO R }}=\left[\begin{array}{cc}
\tilde{\mathbf{U}}_{1}^{T} \cdot \mathbf{E}_{1} \cdot \tilde{\mathbf{U}}_{1} & 0 \\
0 & \mathbf{U}_{2}^{T} \cdot \mathbf{E}_{2} \cdot \mathbf{U}_{2}
\end{array}\right]=\left[\begin{array}{cc}
\tilde{\mathbf{C}} & 0 \\
0 & \tilde{\mathbf{L}}
\end{array}\right] \\
\hat{\mathbf{G}}_{\text {SPRIM_IOPO R }}=\left[\begin{array}{cc}
\tilde{\mathbf{U}}_{1}^{T} \cdot \mathbf{G}_{1} \cdot \tilde{\mathbf{U}}_{1} & \tilde{\mathbf{U}}_{1}^{T} \cdot \mathbf{A}_{1} \cdot \mathbf{U}_{2} \\
-\mathbf{U}_{2}^{T} \cdot \mathbf{A}_{1}^{T} \cdot \tilde{\mathbf{U}}_{1} & \mathbf{U}_{2}^{T} \cdot \mathbf{G}_{2} \cdot \mathbf{U}_{2}
\end{array}\right]=\left[\begin{array}{cc}
\tilde{\mathbf{G}} & \tilde{\mathbf{A}} \\
-\tilde{\mathbf{A}}^{T} & \tilde{\mathbf{R}}
\end{array}\right] \\
\hat{\mathbf{B}}_{\text {SPRIM_IOPO R }}=\left[\begin{array}{c}
\tilde{\mathbf{U}}_{1}^{T} \cdot \mathbf{A}_{\mathrm{I}} \\
0
\end{array}\right]=\left[\begin{array}{c}
\tilde{\mathbf{B}} \\
0
\end{array}\right]
\end{gathered}
$$

The advantage of the SPRIM method is conserved because the nature of reduce state variables are conserved:

$$
\left[\begin{array}{c}
\tilde{\mathbf{v}}_{n}(s) \\
\tilde{\mathbf{i}}_{l}(s)
\end{array}\right]=\left[\begin{array}{c}
\tilde{\mathbf{U}}_{1} \cdot \mathbf{v}_{N}(s) \\
\mathbf{U}_{2} \cdot \mathbf{i}_{L}(s)
\end{array}\right]
$$

Also, we have circuit equations in frequency domain:

$$
\left\{\begin{array}{l}
\left(s \cdot\left[\begin{array}{cc}
\widetilde{\mathbf{C}} & 0 \\
0 & \tilde{\mathbf{L}}
\end{array}\right]+\left[\begin{array}{cc}
\tilde{\mathbf{G}} & \tilde{\mathbf{A}} \\
-\tilde{\mathbf{A}}^{T} & \tilde{\mathbf{R}}
\end{array}\right]\right) \cdot\left[\begin{array}{c}
\widetilde{\mathbf{v}}_{n}(s) \\
\tilde{\mathbf{i}}_{l}(s)
\end{array}\right]=\left[\begin{array}{c}
\widetilde{\mathbf{B}} \cdot \mathbf{u}(s) \\
0
\end{array}\right] \\
\mathbf{y}(s)=\left[\begin{array}{ll}
\widetilde{\mathbf{B}} & 0
\end{array}\right]^{T} \cdot\left[\begin{array}{c}
\widetilde{\mathbf{v}}_{n}(s) \\
\tilde{\mathbf{i}}(s)
\end{array}\right]
\end{array}\right.
$$

By eliminating the auxiliary variables $\tilde{\mathbf{i}}_{l}$, a second-order formulation of the system (26) is given by:

$$
\left\{\begin{array}{l}
{\left[s \cdot \widetilde{\mathbf{C}}+\widetilde{\mathbf{G}}+\tilde{\mathbf{A}} \cdot(\tilde{\mathbf{R}}+s \cdot \tilde{\mathbf{L}})^{-1} \cdot \tilde{\mathbf{A}}^{T}\right] \cdot \widetilde{\mathbf{v}}_{n}(s)=\widetilde{\mathbf{B}} \cdot \mathbf{u}(s)} \\
\mathbf{y}(s)=\widetilde{\mathbf{B}}^{T} \cdot \tilde{\mathbf{v}}_{n}(s)
\end{array}\right.
$$

In equation (27), the block of capacitor and resistance are well separated but not the case of RL elements which is under inversion operation. In order to synthesize equation (27) into a circuit, we need to separate RL elements by these transformations below.

Let's notice that the matrix $\tilde{\mathbf{L}}$ is symmetric positive definite so we can use Cholesky factorization as:

$$
\tilde{\mathbf{L}}=\mathbf{L}_{\text {chol }}^{T} \cdot \mathbf{L}_{\text {chol }}
$$

so we have:

$$
(\widetilde{\mathbf{R}}+s \cdot \tilde{\mathbf{L}})^{-1}=\left(\tilde{\mathbf{R}}+s \cdot \mathbf{L}_{\text {chol }}^{T} \cdot \mathbf{L}_{\text {chol }}\right)^{-1}=\mathbf{L}_{\text {chol }}^{-1} \cdot\left(\mathbf{L}_{\text {chol }}^{-1} \cdot \widetilde{\mathbf{R}} \cdot \mathbf{L}_{\text {chol }}^{-1}+s \cdot \mathbf{I d}\right)^{-1} \cdot \mathbf{L}_{\text {chol }}^{-1} T
$$

With eigen-decomposition to eigenvalues and eigenvectors, we have:

$$
\mathbf{L}_{\text {chol }}^{-1} \cdot \tilde{\mathbf{R}} \cdot \mathbf{L}_{\text {chol }}^{-1}=\mathbf{V} \cdot \mathbf{Z}_{\text {diag }} \cdot \mathbf{V}^{T}
$$

where $\mathbf{Z}_{\text {diag }}$ is diagonal matrix of eigenvalues and a full matrix $\mathbf{V}\left(\mathbf{V} \cdot \mathbf{V}^{\mathbf{T}}=\mathbf{I d}\right)$ whose columns are the corresponding eigenvectors. So we have:

$$
\left(\mathbf{L}_{\text {chol }}^{-1} \cdot \tilde{\mathbf{R}} \cdot \mathbf{L}_{\text {chol }}^{-1}+s \cdot \mathbf{I d}\right)^{-1}=\left(\mathbf{V} \cdot \mathbf{Z}_{\text {diag }} \cdot \mathbf{V}^{T}+s \cdot \mathbf{V} \cdot \mathbf{V}^{T}\right)^{-1}=\mathbf{V} \cdot\left(\mathbf{Z}_{\text {diag }}+s \cdot \mathbf{I d}\right)^{-1} \cdot \mathbf{V}^{T}
$$

From (29) to (31), we have:

$$
\tilde{\mathbf{A}} \cdot(\tilde{\mathbf{R}}+s \cdot \tilde{\mathbf{L}})^{-1} \cdot \tilde{\mathbf{A}}^{T}=\underbrace{\tilde{\mathbf{A}} \cdot \mathbf{L}_{\text {chol }}^{-1} \cdot \mathbf{V}}_{\mathbf{X}} \cdot\left(\mathbf{Z}_{\text {diag }}+s \cdot \mathbf{I d}\right)^{-1} \cdot \underbrace{\mathbf{V}^{T} \cdot \mathbf{L}_{\text {chol }}{ }^{T} \cdot \tilde{\mathbf{A}}^{T}}_{\mathbf{X}^{T}}
$$

Finally, we can rewrite (27) in the new form:

$$
\left\{\begin{array}{l}
\left.s \cdot \widetilde{\mathbf{C}}+\widetilde{\mathbf{G}}+\mathbf{X} \cdot\left[\begin{array}{ccc}
\frac{1}{s+z_{11}} & \cdots & 0 \\
\vdots & \ddots & \vdots \\
0 & \cdots & \frac{1}{s+z_{N N}}
\end{array}\right] \cdot \mathbf{X}^{T}\right] \cdot \widetilde{\mathbf{v}}_{n}(s)=\widetilde{\mathbf{B}} \cdot \mathbf{u}(s) \\
\mathbf{y}(s)=\widetilde{\mathbf{B}}^{T} \cdot \widetilde{\mathbf{v}}_{n}(s)
\end{array}\right.
$$

The equation (33) eliminates all mutual inductances and all the block with nature of RLC are appeared. It is then possible to synthesize to a new circuit equivalent from (33). In the next section, we will present this synthesis method.

\section{Equivalent circuit synthesis method}




\subsection{Foster synthesis for SISO}

This section describes the Foster synthesis method, which was developed in the 1930s by Foster and Cauer (Guillemin, 1959) and involves realization based on the system's transfer function. Let's note that there are two basic approaches to solve the circuit: nodal analysis (using Kirchhoff's current law (KCL)) or mesh analysis approach (Kirchhoff's voltage law (KVL)). The equation (33) represented nodal method with the admittance matrix $\mathbf{Y}$ inside the brackets on the left of equation. The value $Y_{i j}(s)$ of the ith row and the $j t h$ column of the admittance matrix $\mathbf{Y}$ is:

$$
Y_{i j}(s)=s \cdot \tilde{c}_{i j}+\tilde{g}_{i j}+\sum_{k=1}^{m} \frac{x_{i k} \cdot x_{j k}}{s+z_{k k}}
$$

The Foster realization (Guillemin, 1959) converts each term in (34) into the corresponding circuit block with $\mathrm{R}, \mathrm{L}, \mathrm{C}$ components, and connects these blocks in the final nestlist which is shown in Figure 1

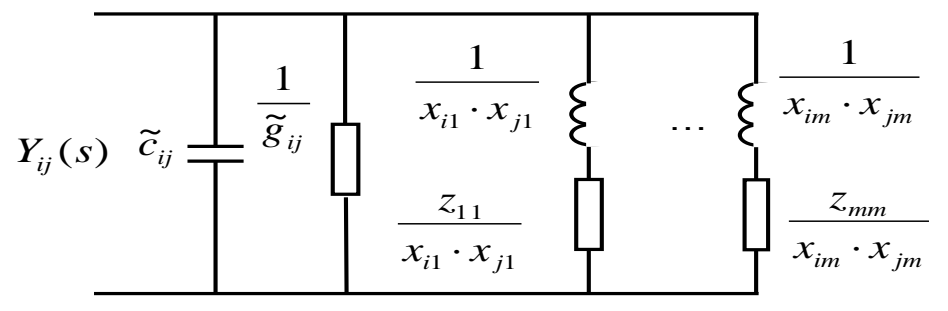

Figure 1. Foster realization for SISO network

The original method is used for two terminal circuit synthesis or single-input-single-output (SISO) system. However, in your case, we need to adapt this method for a more complicated case: the multi-input-multi-output (MIMO) network.

\subsection{RLMC circuit synthesis (RLMCSYN) for MIMO}

Normally, from the topology of the circuit, we can construct the nodal admittance matrix (Ling, 1987). In the case of circuit synthesis, we do the inverse construction; it means that we want to know the circuit topology from the nodal admittance matrix $\mathbf{Y}$. These Kirchhoff's laws (KCL and KVL) give us that the sum of currents entering a node in the circuit is zero, and the sum of voltages around a closed loop starting and ending at a node is also zero and thereby determine the elements of the admittance matrix. The $\mathbf{Y}$ matrix diagonal elements $\mathbf{Y}_{11}$, $\mathrm{Y}_{22} \ldots \mathrm{Y}_{\mathrm{NN}}$ are called the self-admittances at the nodes, and each equals the sum of all the admittances terminating on the node identified by the repeated subscripts. The other admittances are the mutual admittances of the nodes, and each equals the negative of the sum of all admittances connected directly between the nodes identified by the double subscripts (Ling, 1987). The admittance matrix $\mathbf{Y}$ is typically a symmetric matrix as $Y_{i j}=Y_{j i}$.

Based on the construction of nodal admittance matrix in the formula (Ling, 1987):

$$
\begin{aligned}
& Y_{i j_{-} \text {node }}(s)=-Y_{i j_{\text {_branch }}}(s) \\
& Y_{i i_{-} \text {node }}(s)={ }^{n b_{-} \text {branch_connected }} \sum_{j=1} Y_{i j_{-} \text {branch }}(s)
\end{aligned}
$$

We can realize to a RLC circuit shown in Figure 2 from the nodal admittance matrix $\mathbf{Y}$ in (33).We already had the value of nodal admittance and we need to calculate the corresponding branch elements. Starting at node $i$ and node $j(i \neq j)$, the elements connected between two nodes give us the value $Y_{i j}$ and Foster realization can be applied to form the netlist containing capacitor, resistor and PEEC element in parallel. As in (35), the elements connected from node $i$ to the ground (reference potential) is the sum of the nodes connected to the node $i$ and the equivalent circuit is illustrated in Figure 2. When we compute the nodal admittance matrix in Figure 2 by using the formula (35), we will obtain the matrix $\mathbf{Y}$ as in (33), the realization is then correct.

We realize that there are so many parallel branches RL in the synthesized circuit ( $m$ branches between each node which presents the mutual effect). In order to reduce this number, the "vector fitting" method (Gustavsen, et al., 1999; Schilders et al., 2008) is applied. This method has been proved to be efficient for multiport circuits with thousand branches (Deschrijver et al., 2008). The second order reductions (vector fitting method) approximate the equation (34) by least square approximation:

$$
Y_{i j}(s)=s \cdot \tilde{c}_{i j}+\tilde{g}_{i j}+\sum_{k=1}^{m} \frac{x_{i k} \cdot x_{j k}}{s+z_{k k}} \approx \sum_{n=1}^{P} \frac{c_{i j n}}{s-a_{n}}+\tilde{g}_{i j}+s \cdot \tilde{c}_{i j}
$$

In the equation (36) we have $P<<m$ that means after the realization the number of parallel branches RL is then reduced. In the scope of this research, only basic vector fitting is used to reduce the number of circuit elements. This method cannot give a network with all positive R \& L parameters. In future research, a modified version should be implemented (Deschrijver et al., 2007; Tommasi et al., 2011; De Magistris et al., 2011) to ensure physical reliability and also accuracy. 


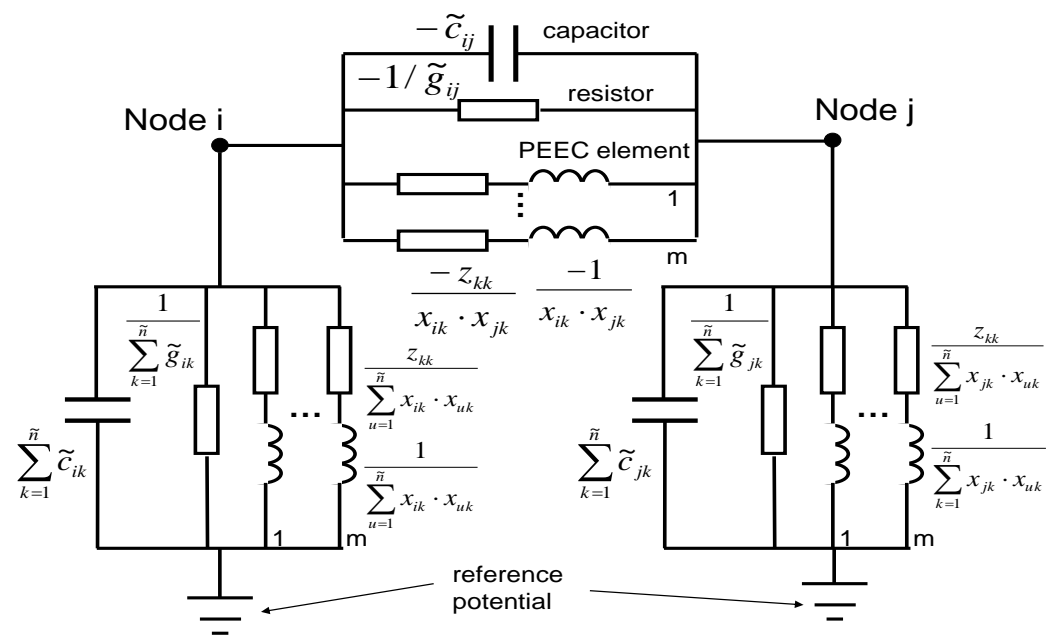

Figure 2. PEEC realization for MIMO network

\section{Numerical results}

In order to validate our method, we compared the frequency response of an EMC filter modelled in the InCa3D software based on PEEC method (Clavel et al., 2002) with the result generated by our synthesis method.

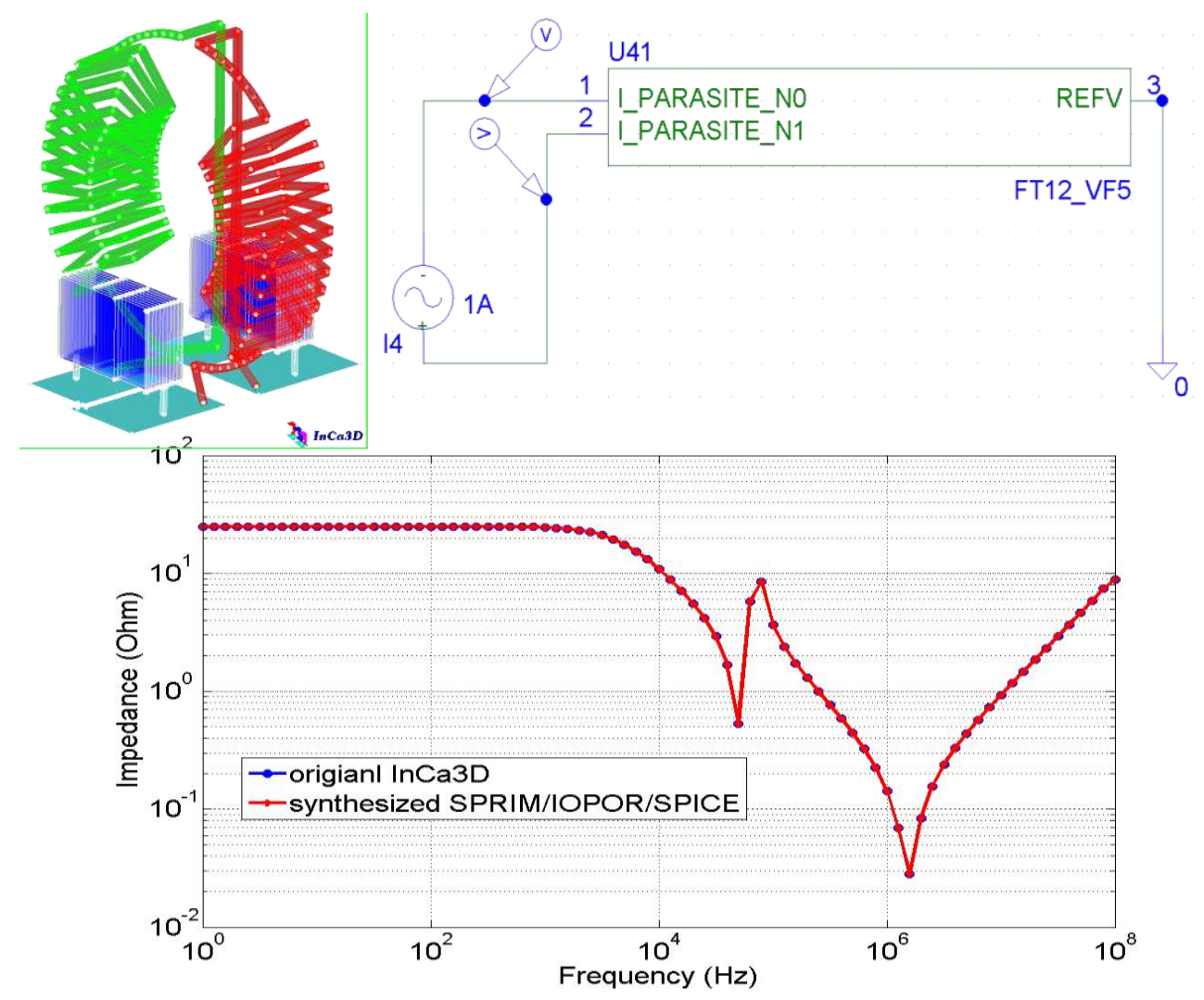

Figure 3. EMC filter modelled in InCa3D (left above), synthesized circuit exported to SPICE (right above), and the comparison of two results (below)

The original equivalent circuit of EMC filter created by PEEC method has 711 nodes 5 capacitors, 4767 resistors, 4765 inductances and 4762*4762 mutual inductances (Figure 3). We take the frequency response calculated by classical PEEC in this software as reference. The frequency range of interest is from $1 \mathrm{~Hz}$ to $100 \mathrm{MHz}$.

By the implementation of the compression algorithm FMM method, up to $85 \%$ of PEEC matrix is compressed. In comparison with InCa3D as reference, the maximal error relative of frequency response is $1.3 \%$. 
Experience from the author of SPRIM method (Freund, 2004) showed that for a multiport PEEC circuit, SPRIM approach give a better approximation than PRIMA method due to the choice of real expansion point different than zero. However, the choice of expansion points or size of Krylov subspace is an open problem. This area of research has barely been touched upon and the problems above still remain open.

The author (Grimme, 1997) suggested that the poles of the models (peak point in frequency response), $\mathrm{f}_{\text {min }}$ and $\mathrm{f}_{\max }$ could have large error, so we have choiced two expansion points in the middle of these points in this case to reduce the error in the reduced model. We generated a model order reduction by multipoint expansion SPRIM/IOPOR method at two real expansion points $\mathrm{s}_{0}=2 \pi \times 10^{5}, \mathrm{~s}_{1}=2 \pi \times 8 \times 10^{7}$ and 7 matching moments for each point. The optimal choice of expansion point or the number of moment matching (q) is an open problem (Silveira et al., 2006; Villena et al., 2009) and need to be considered in further research of MOR framework.

Let's notice that the size of reduced system impact directly to the number of nodes and elements of synthesis circuit. In this case, this choice gives us an acceptable result and small reduced model.

With our choice of $q=7$, after the first run of our new RLMCSYN synthesis method (without the second reduction of RL elements by vector fitting method in section 2), we obtain a circuit of 18 nodes and 4590 inductance branches without any mutual inductance. The synthesis circuit is imported in PSPICE software via a netlist representation (Figure 3). We compared the frequency response obtained in PSPICE with our reference; the maximal relative error is less than $13 \%$.

Because of the nature of inductive PEEC methods, we have a lot of inductance elements in synthesized circuit. In order to reduce these elements, the vector fitting in section 3.2 is used. The error of vector fitting method depends on the number of branch RL as in the Table I and Figure 4.

In Figure 4, the more number of RL branch is kept, the more accurate result we have. In low frequency the inductive effects is negligible while in high frequency, the behaviour of synthesis circuit depends on the number of RL elements. Consequently, the simple circuit with $2 \mathrm{RL}$ branch is good enough in low frequency but has large error in high frequency.

Table I. Reduction by vector fitting method

\begin{tabular}{lcccc}
\hline \hline No of branch RL & 2 & 4 & 6 & 7 \\
\hline No of inductance elements & 612 & 1224 & 1836 & 2142 \\
\hline
\end{tabular}

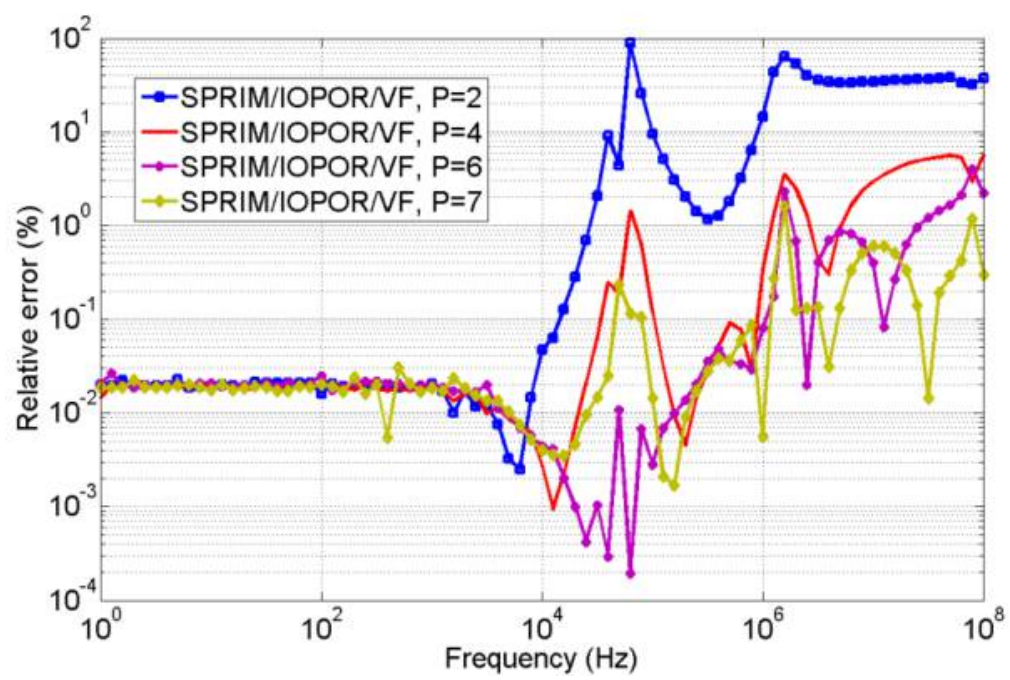

Figure 4. Relative error of vector fitting method

The constraint between number of elements in synthesized circuit and precision makes us choosing the number of branch RL is 6 which makes the result circuit having 18 nodes and 1836 RL elements (less than 5\% relative error).

\section{Conclusion}

In this paper, a framework for realizing reduced mathematical models obtained by Krylov subspace based MOR methods into RLC netlists was proposed in compression matrix context. SPRIM method is used to generate reciprocal macro-models of multiport RLMC circuit and IOPOR technique preserve the input and output structures in the reduced-order systems. A novel RLMC circuit synthesis method (RLMCSYN) is then proposed 
to generate a pure RLC equivalent circuit, passivity preserving, accurate modelling of the original interconnect circuit. In order to reduce the number of RLC elements, the vector fitting is used as a second reduction.

By using a matrix compression algorithm with our synthesis technique, the large, complex problem can be treated in time-domain simulation in any universal temporal simulation tools.

Let's notice that the choice of good MOR method impact strongly in the final synthesis netlist size. Future research will investigate on the direct use of ROM state space equation in comparison with our approach.

\section{Acknowledgment}

This research is funded by Vietnam National Foundation for Science and Technology Development (NAFOSTED) under grant number 102.01-2014.39.

\section{References}

Ardon, V., Aime, J., Chadebec, O., Clavel, E., Guichon, J-M. and Vialardi, E. (2010), "EMC Modeling of an Industrial Variable Speed Drive with an Adapted PEEC Method", IEEE Transactions on Magnetics, Vol. 46 No. 8, pp. 2892-98.

Clavel, E., Roudet, J., Foggia, A. (2002), "Electrical Modeling of Transformer Connecting Bars”, IEEE Transactions on Magnetics, Vol. 38 No. 2, pp. 1378-82.

Chung-Wen, H., Ruehli, A. E. and Pierce, A. B. (1975), "The modified nodal approach to network analysis", IEEE Transactions on Circuits and Systems, Vol. 22 No. 6, pp. 504-09.

Davis, T. A. (2006), "Direct Methods for Sparse Linear Systems", SIAM, Philadelphia, PA.

Deschrijver, D., Haegeman, B., Dhaene, T. (2007), "Orthonormal vector fitting: A robust macromodeling tool for rational approximation of frequency domain responses", IEEE Transactions on advanced packaging, Vol. 30 No. 2, pp. 216-25.

Deschrijver, D., Mrozowski, M., Dhaene, T., De Zutter, D. (2008) "Macromodeling of multiport systems using a fast implementation of the vector fitting method", IEEE Microwave and Wireless Components Letters, Vol. 18 No. 6, pp. 383-85.

De Magistris, M. and Nicolazzo, M. (2011), "On the concretely passive realization of reduced circuit models based on convex constrained positive real fractions identification", 15th IEEE Workshop on Signal Propagation on Interconnects (SPI), Naples, Italy.

Ekman, J., Antonini, G. and Orlandi, A. (2004), "Full-wave time domain PEEC formulation using a modified nodal analysis approach", EMC Europe 2004: International Symposium on Electromagnetic Compatibility, Eindhoven, Netherlands.

Feng, L., Korvink, J.G., Benner, P. (2015), "A Fully Adaptive Scheme for Model Order Reduction Based on Moment-matching", IEEE Transactions on Components, Packaging and Manufacturing Technology, Vol. 15 No. 12, pp.1872 - 84.

Ferranti, F., Nakhla, M.S., Antonini, G., Dhaene, T., Knockaert, L. and Ruehli, A.E. (2011),"Multipoint full-wave model order reduction for delayed PEEC models with large delays", IEEE Transactions on Electromagnetic Compatibility, Vol. 53 No.4, pp. 959-67.

Freund, R.W. (2004), "SPRIM: structure-preserving reduced-order interconnect macromodeling," Tech. Dig. IEEE/ACMInternational Conference on Computer-Aided Design, IEEE Computer Society Press, Los Alamitos, California, pp. 80-87.

Grimme, E.J. (1997), “Krylovprojection methods for model reduction”, Thesis Diss. University of Illinois, USA.

Guillemin, E. A. (1959), "Synthesis of passive networks”, John Wiley.

Gustavsen, B. and Semlyen, A. (1999), "Rational approximation of frequency domain responses by vector fitting", IEEE Transaction on Power Delivery, Vol. 14 No. 3, pp. 1052-61.

Ionutiu, R., and Rommes, J. (2009), "CASA-Report 09-28 September 2009", Eindhoven University of Technology, Netherlands.

Kaufmann, C. (2010), "Identification of Electrical Circuits for Realization of Sparsity Preserving Reduced Order Models", Ph.D Theses, University of Wuppertal, Germany.

Ling, D. and Ruehli, A. (1987), “Circuit Analysis, Simulation and Design-Advances in CAD for VLSI”, Elsevier Science Publisher.

Nguyen, T-S., Guichon, J-M., Chadebec, O. and Meunier, G. (2012), "Equivalent circuit synthesis method for reduced order models of large scale inductive PEEC circuits", Proceedings of CEFC, Oita, Japan.

Odabasioglu, A., Celik, M. and Pileggi, L. (1997), "PRIMA: Passive reduced order interconnect macromodeling algorithm", IEEE/ACM International Conference on Computer Aided-Design, San Jose, USA, pp. 58-65.

Rasekh, E. and Dounavis, A. (2012), "Multiorder Arnoldi Approach for Model Order Reduction of PEEC Models With Retardation", IEEE Transactions on Components, Packaging and Manufacturing Technology, Vol. 2 No. 10, pp. 1629 - 36

Ruehli, A.E. (1974), "Equivalent circuit models for three dimensional multiconductor systems", IEEE Transaction on Microwave Theory and Techniques, Vol. 22 No. 3, pp. 216-21. 
Silveira, L.M. and Phillips, J.R. (2006), "Resampling plans for sample point selection in multipoint model-order reduction", IEEE Transactions on Computer-Aided Design of Integrated Circuits and Systems, Vol. 25 No.12, pp. 2775-83.

Schilders, W.H.A, Van der Vorst, H.A. and Rommes, J. (2008), "Model Order Reduction: Theory, Research Aspects and Applications", Mathematics in Industry Vol. 13, Springer Berlin Heidelberg, ISBN: 978-3-540-78840-9 (Print) 978-3-540-78841-6 (Online).

Tommasi, L.D, De Magistris, M., Deschrijver, D. and Dhaene, T. (2011), "An algorithm for direct identification of passive transfer matrices with positive real fractions via convex programming", International Journal of Numerical Modelling: Electronic Networks, Devices and Fields, Vol. 24 No. 4, pp. 375-86.

Villena, J.F. and Silveira, .L.M. (2009), "ARMS-automatic residue-minimization based sampling for multi-point modeling techniques", 46th ACM/IEEE Design Automation Conference (DAC '09), San Francisco, USA.

Wollenberg, G. and Kochetov, S.V. (2011), "PEEC method: circuit models for electromagnetic field problems", COMPEL - The international journal for computation and mathematics in electrical and electronic engineering, Vol. 30 No. 4, pp.1283 - 95.

Wolf, T., Panzer, H. and Lohmann, B. (2011), "Gramian-based error bound in model reduction by Krylov subspace methods" 18th IFAC World Congress, Milano, Italy.

Yang, F., Zeng, X., Su, Y. and Zhou, D. (2008), "RLC equivalent circuit synthesis method for structure-preserved reduced-order model of interconnect in VLSI", Communications in Computational Physics, Vol. 3 No. 2, pp. 376-96.

Zhang, Z, Xie, X., Li, L., Xiao, D. and He, W. (2014), "Super-fast multipole method for power frequency electric field in substations", COMPEL: The International Journal for Computation and Mathematics in Electrical and Electronic Engineering, Vol. 33 Iss: $1 / 2$, pp.594 610 .

\section{Authors affiliation :}

- Trung-Son Nguyen is with Vietnam Fire and Rescue Police Department Hanoi, Viet Nam.

- Tung Le Duc is with Hanoi University of Science and Technology - School of Electrical Engineering Hanoi Viet Nam and Hanoi University of Science and Technology Hanoi Viet Nam.

- Son Thanh Tran is with Electric Power University, Hanoi, Viet Nam

- Jean-Michel Guichon and Olivier Chadebec are with Univ. Grenoble Alpes, CNRS, Grenoble INP, G2Elab, Grenoble, France 